\title{
Acute kidney injury associated with immune checkpoint inhibitor therapy: incidence, risk factors and outcomes
}

\author{
Alejandro Meraz-Muñoz (D) , ${ }^{1}$ Eitan Amir, ${ }^{2}$ Pamela Ng, ${ }^{3}$ Carmen Avila-Casado, ${ }^{4}$ \\ Claire Ragobar, ${ }^{1}$ Christopher Chan, ${ }^{1}$ Joseph Kim, ${ }^{1}$ Ron Wald,${ }^{5}$ Abhijat Kitchlu (1) ${ }^{1}$
}

To cite: Meraz-Muñoz A, Amir E, Ng P, et al. Acute kidney injury associated with immune checkpoint inhibitor therapy: incidence, risk factors and outcomes. Journal for ImmunoTherapy of Cancer 2020;8:e000467. doi:10.1136/ jitc-2019-000467

- Additional material is published online only. To view please visit the journal online (http://dx.doi.org/10.1136/jitc2019-000467).

Accepted 26 May 2020

Check for updates

(c) Author(s) (or their employer(s)) 2020. Re-use permitted under CC BY-NC. No commercial re-use. See rights and permissions. Published by BMJ.

${ }^{1}$ Department of Medicine, Division of Nephrology, University Health Network, Toronto, Ontario, Canada ${ }^{2}$ Department of Medicine, Division of Medical Oncology and Hematology, University Health Network, Toronto, Ontario, Canada

${ }^{3}$ Department of Pharmacy, University Health Network, Toronto, Ontario, Canada ${ }^{4}$ Department of Pathology, University Health Network, Toronto, Ontario, Canada ${ }^{5}$ Department of Medicine, Division of Nephrology, Unity Health, Toronto, Ontario, Canada

Correspondence to

Dr Abhijat Kitchlu;

Abhijat.kitchlu@uhn.ca

\section{ABSTRACT}

Background Immune checkpoint inhibitors (ICPi) are a novel and promising anti-cancer therapy. There are limited data on the incidence, risk factors and outcomes of acute kidney injury (AKI) in patients receiving ICPi.

Methods We conducted a cohort study of patients receiving ICPi at our center between 2010 and 2017 via electronic health record. The primary outcome was AKI (increase of $>50 \%$ from baseline serum creatinine $(\mathrm{sCr}))$. Risk factors for AKI were assessed using logistic regression. Survival among those with and without AKI was compared using the Kaplan-Meier method.

Results Among 309 patients on ICPi, 51 (16.5\%) developed AKI (Kidney Disease Improving Global Outcomes (KDIGO) stages 1: 53\%, 2: 22\%, 3: 25\%). AKI was associated with other immune-related adverse events (IRAE) (OR 3.2, 95\% Cl 1.6 to 6; $\mathrm{p}<0.001$ ), hypertension (OR 4.3, 95\% Cl 1.8 to $6.1 ; p<0.001$ ) and cerebrovascular disease (OR 9.2; 95\% Cl 2.1 to 40; $p<0.001$ ). Baseline $\mathrm{sCr}$, cancer, and ICPi type was not associated with AKI. Use of angiotensin-converting enzyme inhibitors/angiotensinreceptor blockers (OR 2.9; 95\% $\mathrm{Cl} 1.5$ to $5.7 ; \mathrm{p}=0.002$ ), diuretics (OR 4.3; 95\% $\mathrm{Cl} 1.9$ to $9.8 ; \mathrm{p}<0.001$ ), and corticosteroid treatment (OR 1.9; $95 \% \mathrm{Cl} 1.1$ to 3.6 ; $\mathrm{p}=0.03$ ) were associated with AKI. In the multivariable analysis, AKI was associated only with other IRAE (OR 2.82; $95 \% \mathrm{Cl} 1.45$ to $5.48 ; \mathrm{p}=0.002$ ) and hypertension (OR 2.96; $95 \% \mathrm{Cl} 1.33$ to $6.59 ; \mathrm{p}=0.008)$. AKI was not associated with increased risk of mortality (HR 1.1; $95 \% \mathrm{Cl}: 0.8$ to $1.6 ; p=0.67$ ). ICPi nephrotoxicity was attributed via biopsy or nephrologist assessment in 12 patients (six interstitial nephritis, two membranous nephropathy, two minimal change disease, and two thrombotic microangiopathy). Subsequent doses of ICPi were administered to 12 patients with prior AKI, with one (8.3\%) having recurrent AKI. Conclusion AKI is a common complication in patients receiving ICPi treatment. The development of other IRAE and previous diagnosis of hypertension were associated with increased AKI risk. AKI was not associated with worse survival. Distinguishing kidney IRAE from other causes of AKI will present a frequent challenge to oncology and nephrology practitioners. Kidney biopsy should be considered to characterize kidney lesions and guide potential therapy.

\section{INTRODUCTION}

Immune checkpoint inhibitors (ICPi) are a novel and promising anti-cancer therapy.
This novel class of drugs are humanized antibodies that inhibit down-regulatory immune pathways (including cytotoxic T-lymphocyte antigen 4 (CTLA-4) and programmed cell death 1 (PD-1) or its ligand, (PD-L1)) with the objective of enhancing the antitumor immune response. ${ }^{1}$ These therapies are used increasingly in a wide spectrum of cancers such as melanoma, lung cancer, urothelial and renal cell carcinomas, among others. $^{2}$ However, by increasing the activity of the immune system, ICPi can precipitate inflammatory side effects, which are termed immune-related adverse events (IRAE). These most commonly affect the skin, gastrointestinal tract, liver and endocrine system, but may involve any organ system, including the kidneys. ${ }^{2}{ }^{3}$ A spectrum of kidney IRAE have been described in case reports and smaller series, including, acute interstitial nephritis (AIN), acute tubular necrosis (ATN), and less commonly, glomerular disease. ${ }^{4-8}$

Data on the incidence and characteristics of nephrotoxicity from ICPi are limited and derived largely from small case series and oncologic studies which reported on kidney safety outcomes using the Common Terminology Criteria for Adverse Effects (CTCAE) for an acute kidney injury (AKI) diagnosis. The incidence of nephrotoxicity with these agents has been reported to be as low as $2 \%$ with a single agent nivolumab and up to $4.5 \%$ when a combination of ipilimumab plus nivolumab were used. These estimates were obtained from the results of phase I, II, and III cancer trials and case series that provided data on kidney adverse events. ${ }^{9-12}$ However, given that patients undergoing systemic treatment for cancer are at high risk for AKI via multiple mechanisms (eg, volume depletion, obstructive nephropathy, hypercalcemia, and so on), establishing ICPi-related nephrotoxicity versus other causes of AKI may be challenging. 
Implicating a therapy-related cause of AKI may also have important consequences for ongoing oncologic treatment and outcomes. Only a minority of patients with cancer who develop AKI will receive nephrology assessment and even fewer may undergo kidney biopsy to definitively establish an etiology. At present, there are minimal data to identify risk factors for AKI, specifically ICPi-related nephrotoxicity, in patients receiving ICPi. Thus far, risk factors such as the presence of other IRAE, co-prescription of other drugs associated with AIN (eg, proton pump inhibitors (PPI) and antibiotics and nonsteroidal anti-inflammatory drugs (NSAIDs)) have been postulated; however, limited data exists to support this. We sought to describe the incidence of AKI in all patients who received ICPi therapy (ipilimumab, nivolumab, or pembrolizumab) at a single center. Second, we assessed patient-level risk factors for AKI and the impact of AKI on clinical outcomes.

\section{METHODS}

\section{Study design and setting}

We conducted a retrospective cohort study of patients aged $\geq 18$ years who received at least one dose of ipilimumab, nivolumab or pembrolizumab at a large academic regional cancer center (Princess Margaret Cancer Centre), in Toronto, Canada. We identified patients receiving these agents between January 1, 2010, and January 1, 2017, via an institution-wide electronic order entry system for oncologic treatments. Patients with endstage kidney disease (ESKD) on dialysis or with previous kidney transplant were excluded (online supplementary figure 1).

\section{Data sources}

Patients who received ICPi therapy were identified using the electronic Oncology Pharmacy Information System (OPIS), which is used to order systemic treatments (including all ICPi) at our center, outside the setting of clinical trials. The following variables related to patient demographics and therapy administration was collected via OPIS: age, sex, weight, cancer type and stage, ICPi type and dose, and therapy start and end dates. Data were then linked to our institutional electronic patient record (EPR) in order to obtain additional baseline characteristics and serum creatinine (sCr) data. The following baseline data was abstracted from the EPR: comorbidities that may influence the development of nephrotoxicity (hypertension, diabetes, congestive heart failure, history of myocardial infarction, liver disease, autoimmune diseases, concomitant cancers, peripheral vascular diseases, cerebrovascular disease, chronic obstructive pulmonary disease or asthma, HIV status), Charlson score, potential nephrotoxic medications received while on ICPi therapy, other IRAEs before or concomitant with the AKI episode, and the last date of available follow-up. A computer algorithm was used to obtain baseline $\mathrm{sCr}$ (ie, the measurement obtained closest to, but prior to the date of first ICPi dose). The algorithm also provided peak sCr values during ICPi therapy as well as during the 90-day period following the last ICPi dose (in this way, we attempted to capture late ICPi-related nephrotoxicity). All sCr data obtained from the algorithm were then reviewed manually and verified.

\section{Outcomes}

The primary outcome was incidence of AKI associated with ICPi therapy. AKI was defined and classified according to the Kidney Disease Improving Global Outcomes (KDIGO) sCr criteria. ${ }^{13}$ We omitted the KDIGO criterion for $\mathrm{sCr}$ change within 7 days (as in this primarily outpatient cohort, many patients may not have had sCr drawn within the 7 days prior to AKI onset). As secondary outcomes, we included the assessment of pre-specified baseline characteristics as risk factors for the development of AKI during ICPi therapy. We also compared mortality in patients who did and did not develop AKI during therapy. Additionally, we reported the cases of nephrologist-confirmed ICPirelated nephrotoxicity and characterized these according to presumed or confirmed kidney lesion (glomerular or interstitial) via kidney biopsy. We considered cases to be nephrologist-confirmed ICPi-related nephrotoxicity only if there was a clear statement from the consulting nephrologist as to the presumptive kidney lesion or an available kidney biopsy report.

\section{Statistical analysis}

Risk factors associated with ICPi-related AKI were assessed using logistic regression. We performed univariate regression to evaluate the relationship between all measured baseline characteristics and AKI episodes. We retained those covariates found to be significantly associated with AKI in a multivariable regression model (limiting the model to four covariates to avoid model over-fit). We reported OR with 95\% CI for each covariate of interest. To assess the association between AKI and subsequent mortality, we performed survival analysis using the Kaplan-Meier method. We reported (an unadjusted) HR for mortality with $95 \%$ CI. Two-sided $p$ values $<0.05$ were considered statistically significant. All analyzes were performed using SPSS V.25 (Armonk, NY).

\section{Post hoc analyses and sensitivity analysis for AKI risk factors}

We further characterized AKI episodes as either sustained (ie, an elevation of $\geq 1.5$ times baseline sCr that persists for $\geq 72$ hours) versus non-sustained (ie, elevation of $\geq 1.5$ times baseline sCr with duration $<72$ hours). In those patients without nephrologist-confirmed ICPi nephrotoxicity, we attempted to ascertain the cause of AKI via chart review and assign a presumptive etiology. We categorized these AKI episodes as 'Possible ICPi nephrotoxicity' versus 'AKI likely from other causes' (eg, pre-renal/hemodynamic, sepsis-related or obstructive AKI). We defined cases of 'Possible ICPi nephrotoxicity' as per the criteria proposed by Gupta et al. ${ }^{14}$ In a sensitivity analysis, we repeated our analysis of risk factors for the development 
Table 1 Baseline characteristics of the cohort

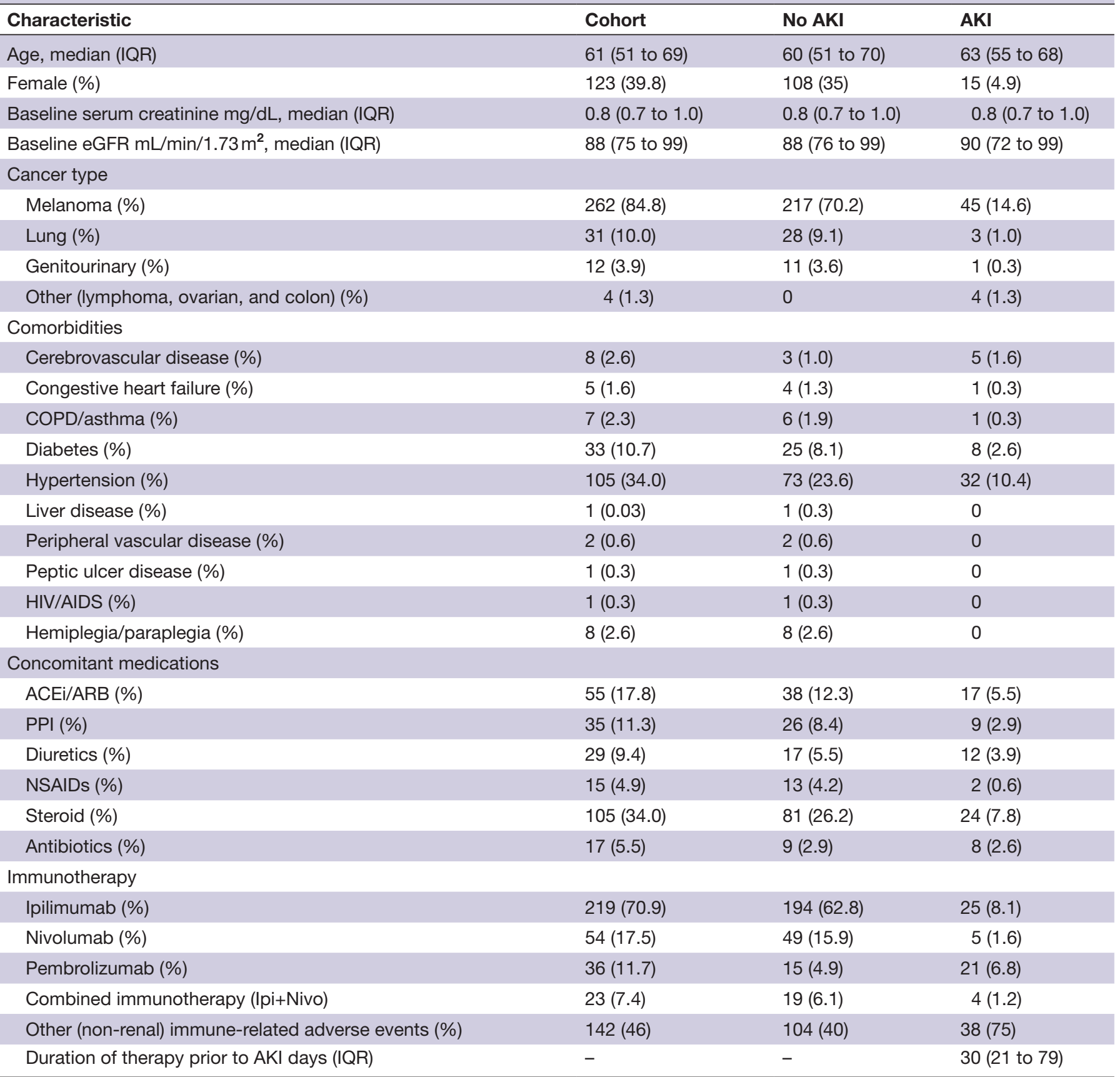

ACEi, angiotensin-converting enzyme inhibitors; AKI, acute kidney injury; ARB, angiotensin-receptor blockers; COPD, chronic obstructive pulmonary disease; eGFR, estimated glomerular filtration rate; Ipi, ipilimumab; Nivo, nivolumab; NSAIDs, non-steroidal antiinflammatory drugs; PPI, proton pump inhibitors.

of AKI, including only the cases of confirmed or possible ICPi nephrotoxicity.

\section{RESULTS}

A total of 354 patients who received at least one dose of ICPi were screened. In all, 45 patient were excluded: 44 had duplicate data and one patient had ESKD (online supplementary figure 1). A total of 309 individual patients who initiated ICPi therapy were included in the study. Among them, 186 (60\%) were male and the median age was 61 years (IQR 51 to 69 ) (table 1 ). The most common cancers included were melanoma 262 $(85 \%)$, lung $31(10 \%)$, and genitourinary cancers 12 (4\%). The most common immunotherapy used was ipilimumab $(70.9 \%)$ alone or in combination. Baseline estimated glomerular filtration rate (eGFR) using the Chronic Kidney Disease Epidemiology Collaboration (CKD-EPI) equation ${ }^{15}$, was 88 (IQR 75 to 99 ) $\mathrm{mL} /$ $\min / 1.73 \mathrm{~m}^{2}$. Hypertension and diabetes mellitus were the most frequent comorbidities in the cohort $(34 \%$ 
Table 2 Characteristics of AKI events

\begin{tabular}{|c|c|}
\hline AKI (any stage) (\%) & $51(16.5)$ \\
\hline AKI stage 1 & $27(53)$ \\
\hline AKI stage 2 & $11(22)$ \\
\hline AKI stage 3 & $13(25)$ \\
\hline Receipt of renal replacement therapy & $1(1.9)$ \\
\hline $\begin{array}{l}\text { Sustained AKI (ie, elevation } \geq 1.5 \text { times baseline } \\
\text { sCr for } \geq 72 \text { hours) (\%) }\end{array}$ & $44(86)$ \\
\hline $\begin{array}{l}\text { Non-sustained AKI (ie, elevation } \geq 1.5 \text { times } \\
\text { baseline sCr for }<72 \text { hours) (\%) }\end{array}$ & $7(14)$ \\
\hline $\begin{array}{l}\text { Biopsy- or nephrologist-confirmed nephrotoxicity } \\
\text { (\%) }\end{array}$ & $12(24)$ \\
\hline
\end{tabular}

Patients with AKI who received ICPi re-challenge 12 (24) (\%)

Patients who developed AKI after re-challenge $\quad 1$ (8.3) (\%)

Presumptive $\mathrm{AKI}$ etiologies among patients with sustained AKI (\%)

\begin{tabular}{ll} 
Biopsy- or nephrologist-confirmed sustained AKI & $12(27)$ \\
Possible ICPi-related AKI & $18(41)$ \\
AKI likely related to other causes (eg, pre-renal/ & $14(32)$ \\
hemodynamic, sepsis-related or obstructive AKI) & \\
\hline
\end{tabular}

AKI, acute kidney injury; ICPi, immune checkpoint inhibitors; sCr, serum creatinine.

and $11 \%$, respectively). Median follow-up was 261 (IQR: 70 to 1072 ) days.

\section{AKI incidence and characteristics}

A total of $54(17.4 \%)$ patients developed AKI or other nephrologist-confirmed nephrotoxicity following initiation of ICPi therapy (table 2). In all, there were 51 AKI episodes $(16.5 \%)$, the majority being of mild-to-moderate severity (KDIGO stages 1: 27 (53\% of AKI episodes), 2: 11 $(22 \%)$, and 3: $13(25 \%))$, and only one patient received acute renal replacement therapy. Median time from ICPi initiation to AKI was 30 (IQR 21 to 79) days. Of patients who experienced nephrotoxicity, 45 of them $(83 \%$ of cases) presented in melanoma patients and 12 (22\% of cases, $7.1 \%$ of total ICPi recipients) had confirmed nephrotoxicity attributed to ICPi (via biopsy or clinical diagnosis from a nephrologist). Kidney lesions and clinical course for patients with nephrologist confirmed ICPi nephrotoxicity is summarized in table 3 . AIN was clinically diagnosed by a nephrologist in five patients and thrombotic microangiopathy (TMA) in one patient. There was one patient with biopsy-confirmed AIN and five patients with other biopsy-confirmed glomerular lesions, including two minimal change disease, two membranous nephropathy and one TMA (online supplementary figures 2-4). Of the 12 patients with biopsy- or nephrologist-confirmed ICPi nephrotoxicity, seven had other non-kidney IRAE (two patients had rash, three had colitis, one had hepatitis, one had myocarditis, one had thyroiditis, and one had other IRAE; two patients had more than one IRAE).

Among patients who survived $>90$ days after the AKI episode $(n=25)$, all stopped ICPi and half received treatment with corticosteroids $(52 \%)$. Full and partial recovery was observed in $22(88 \%)$ and three $(12 \%)$ patients, respectively. Re-challenge with ICPi occurred in 12 patients with nephrotoxicity, with only one $(8.3 \%)$ having recurrent AKI. Of the 12 patients with nephrotoxicity who were re-challenged with ICPi, four had nephrologist-confirmed ICPi-related nephrotoxicity as the cause of initial AKI (two biopsy-confirmed membranous nephropathy (MN) and two AIN), four had possible ICPi-related AKI and four had AKI potentially from other causes. If only patients with nephrologist-confirmed or possible ICPi nephrotoxicity are considered, the proportion with recurrence would be $12.5 \%$ (online supplementary table 1).

At the end of the follow-up, $222(72 \%)$ patients had died. AKI was not associated with increased risk of mortality (HR 1.1; 95\% CI 0.8 to 16 ; $\mathrm{p}=0.67$ ) (figure 1 ).

\section{Risk factors for AKI in patients receiving ICPi}

In the univariable analysis, AKI was associated with the presence of other IRAE (OR 3.2; 95\% CI 1.6 to 6.1; $\mathrm{p}<0.001$ ), diagnosis of pre-existing hypertension (OR 4.3 ; $95 \%$ CI 1.8 to $6.1 ; \mathrm{p}<0.001)$ and history of cerebrovascular disease (OR 9.2; 95\% CI 2.1 to $40.0 ; \mathrm{p}<0.001$ ). Use of angiotensin-converting enzyme inhibitors (ACEi) or angiotensin-receptor blockers (ARB) (OR 2.9; 95\% CI 1.5 to 5.7 ; $\mathrm{p}=0.002$ ), diuretics (OR 4.3 ; $95 \%$ CI 1.9 to 9.8 ; $\mathrm{p}<0.001)$, and corticosteroid treatment (OR 1.9; $95 \% \mathrm{CI}$ 1.1 to 3.6; $\mathrm{p}=0.03$ ) were also associated with AKI. Baseline sCr, eGFR, type of cancer, and ICPi agent were not associated with AKI. In the multivariable analysis, AKI was associated only with the presence of other IRAE (OR 2.82; 95\% CI 1.45 to $5.48 ; \mathrm{p}=0.002$ ) and previous diagnosis of hypertension (OR 2.96; 95\% CI 1.33 to $6.59 ; \mathrm{p}=0.008$ ) (table 4).

\section{Post hoc analyses of presumptive AKI etiologies and sensitivity analysis for AKI risk factors}

We reviewed sCr trends among the patients with AKI and found 44 patients ( $86 \%$ of patients with AKI) experienced a sustained AKI episode (ie, an elevation of $\geq 1.5$ times baseline sCr that persisted for $\geq 72$ hours) (table 2). Apart from those patients with biopsy- or nephrologist-confirmed ICPirelated AKI, 18 patients (41\%) had possible ICPi-related AKI, while 14 (32\%) had AKI likely related to other (nonICPi-related) causes.

In our sensitivity analysis in which we repeated our multivariable logistic regression for AKI risk factors with the exclusion of AKI episodes likely related to other (non-ICPirelated) causes, we found that the presence of other IRAE remained associated with AKI (table 4). The magnitude of the effect was increased with OR 5.59; $95 \%$ CI 2.18 to 14.33 ; $\mathrm{p}<0.001$. In this analysis, pre-existing hypertension, use of 


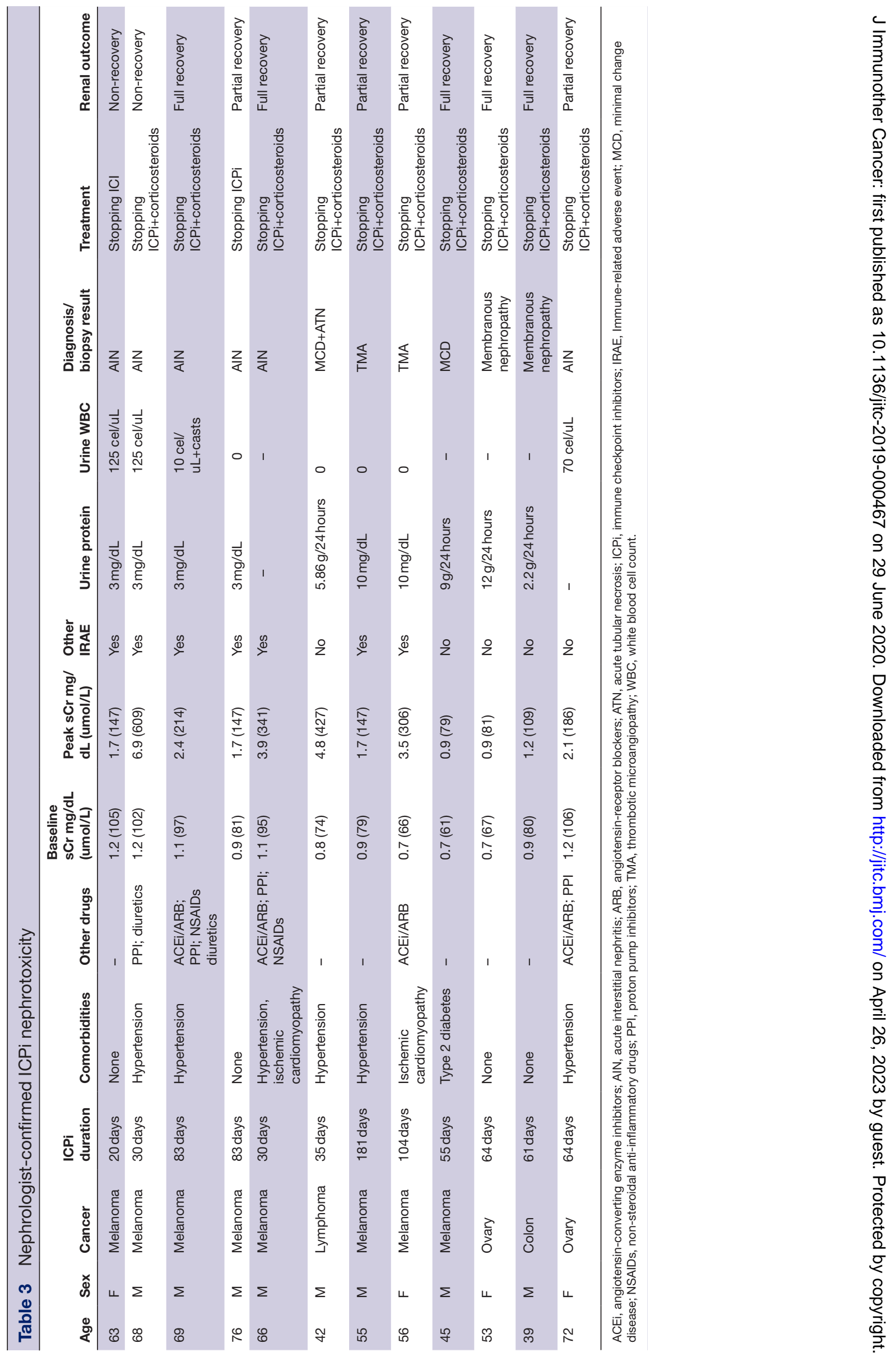




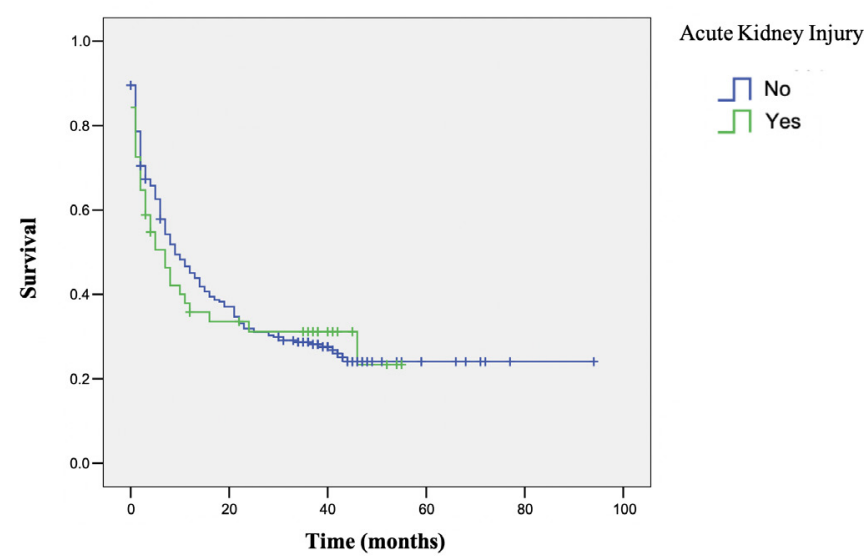

Figure 1 Survival curves for patients with and without AKI during ICPi therapy. Kaplan-Meier curves depicted for patients with no AKI episode during therapy (blue line) versus those who experienced an AKI episode (green line). AKI, acute kidney injury; ICPi, immune checkpoint inhibitors.

ACEi/ARB, and diuretics were not significantly associated with AKI risk.

\section{DISCUSSION}

Our findings demonstrate a considerable burden of AKI among patients treated with ICPi therapy. The substantial incidence of AKI in this population likely reflects a combination of causes of kidney dysfunction, including both ICPi-related nephrotoxicity and the myriad other forms of kidney injury inherent to patients receiving anticancer therapy. ${ }^{16} 17$ The frequency of AKI we observed in this cohort, suggests that oncologists and nephrologists assessing these patients may be faced routinely with discerning kidney IRAE from other causes. The incidence of AKI in our cohort is higher than previously reported from the randomized trials that studied these agents (ie, $2 \%$ to $5 \%$ of ICPi recipients), ${ }^{4578}$ however, may be more in keeping with higher rates $(9.9 \%$ to $29 \%)$ reported in emerging data from conference abstracts ${ }^{18-20}$ and the only other large cohort study of AKI in ICPi recipients. ${ }^{21}$ Seethapathy et al also reported an AKI incidence in ICPi recipients of approximately $17 \%,{ }^{21}$ and this is similar to previously reported incidence of AKI in cancer patients receiving older systemic therapies. ${ }^{22}{ }^{23}$ Interestingly, only a minority of patients had nephrologist-confirmed ICPi nephrotoxicity, despite many patients receiving corticosteroid therapy at the time of AKI (although this may also reflect the presence of other IRAE). Moreover, among the cases of nephrologist-confirmed ICPi nephrotoxicity, half demonstrated glomerular lesions. This may also be due to oncology practitioners being more likely to seek nephrologist consultation for patients with features suggestive of glomerulopathy, such as more rapid kidney function change or high-grade proteinuria.

The existing literature on ICPi nephrotoxicity has predominantly included case series of patients identified following kidney biopsy, and while more specific to kidney IRAE, this may have underestimated the incidence
Table 4 Risk factors for AKI in patients receiving ICPi

\begin{tabular}{|lll}
\hline Variable & OR (95\% Cl) & P value \\
\hline Univariable analysis & & \\
\hline Age & $1.02(0.99$ to 1.04$)$ & 0.17 \\
\hline Female sex & $0.58(0.31$ to 1.10$)$ & 0.1 \\
\hline $\begin{array}{l}\text { Baseline } \mathrm{sCr} \text { (per } 0.1 \mathrm{mg} / \\
\text { dL or } 9 \text { umol/L) }\end{array}$ & $1.02(0.90$ to 1.16$)$ & 0.72 \\
\hline Charlson score & $1.16(1.01$ to 1.33$)$ & 0.042 \\
\hline Other IRAE & $3.19(1.68$ to 6.05$)$ & $<0.001$ \\
\hline Cerebrovascular disease & $9.24(2.13$ to 40.0$)$ & 0.003 \\
\hline Chronic heart failure & $1.27(0.14$ to 11.6$)$ & 0.83 \\
\hline COPD/asthma & $0.68(0.10$ to 4.70$)$ & 0.69 \\
\hline Dementia & $2.58(0.46$ to 14.5$)$ & 0.28 \\
\hline Depression & $1.29(0.41$ to 4.02$)$ & 0.66 \\
\hline Diabetes & $1.78(0.75$ to 4.20$)$ & 0.19 \\
\hline Hypertension & $4.27(2.28$ to 8.01$)$ & $<0.001$ \\
\hline Myocardial infarction & $1.91(0.58$ to 6.26$)$ & 0.29 \\
\hline Rheumatic disease & $1.25(0.14$ to 11.4$)$ & 0.84 \\
\hline ACE/ARBi & $2.90(1.47$ to 5.69$)$ & 0.002 \\
\hline PPI & $1.90(0.83$ to 4.35$)$ & 0.13 \\
\hline Diuretics & $4.34(1.93$ to 9.79$)$ & $<0.001$ \\
\hline NSAIDs & $0.77(0.17$ to 3.52$)$ & 0.74 \\
\hline Steroids & $1.94(1.06$ to 3.57$)$ & 0.033 \\
\hline Antibiotics & $1.22(0.81$ to 1.86$)$ & 0.34 \\
\hline Multivariable analysis & & \\
\hline Other IRAE & $2.82(1.45$ to 5.48$)$ & 0.002 \\
\hline Hypertension & $2.96(1.33$ to 6.59$)$ & 0.008 \\
\hline Use of ACE/ARBi & $1.18(0.51$ to 2.72$)$ & 0.69 \\
\hline Use of diuretics & $1.96(0.78$ to 4.94$)$ & 0.15 \\
\hline Sensitivity* multivariable analysis & \\
\hline Other IRAE & $5.59(2.18$ to 14.33$)$ & $<0.001$ \\
\hline Hypertension & $1.66(0.59$ to 4.65$)$ & 0.34 \\
\hline Use of ACE/ARBi & $1.60(0.54$ to 4.70$)$ & 0.39 \\
\hline Use of diuretics & $2.33(0.68$ to 7.95$)$ & 0.18 \\
\hline Sentity andyis & & \\
\hline
\end{tabular}

*Sensitivity analysis includes only AKI events that were biopsy- or nephrologist-confirmed to be ICPi-related or possible ICPi-related (ie, excluding AKI events likely related to other causes); $n=288$. $\mathrm{ACE}$, angiotensin-converting enzyme; $\mathrm{AKI}$, acute kidney injury; $\mathrm{ARBi}$, angiotensin-receptor blocker inhibitors; COPD, chronic obstructive pulmonary disease; ICPi, immune checkpoint inhibitors; IRAE, immune-related adverse event; NSAIDs, nonsteroidal anti-inflammatory drugs; PPI, proton pump inhibitors.

of nephrotoxicity in these patients. Cortazar et $a t^{t}$ described the histologic features of 13 patients with ICPiassociated nephrotoxicity who underwent kidney biopsy. The most common pathologic lesion encountered was AIN in 12 patients and one with TMA. Similarly, Shirali et $a \tilde{l}$ described the histologic features of six lung cancer patients with ICPi-induced AKI who underwent kidney biopsy, of whom all had AIN. As a result of these initial 
series, AIN was felt to be the predominant form of nephrotoxicity from ICPi. Subsequently, case reports demonstrated other nephrotoxicity in the form of glomerular injury, including MCD, MN, IgA nephropathy, pauciimmune glomerulonephritis, and lupus-like glomerulonephritis. ${ }^{6-8}{ }^{24-26}$ Our cohort adds to the existing data suggesting that glomerular injury may represent a significant proportion of ICPi nephrotoxicity.

Current American Society of Clinical Oncology (ASCO) guidelines for the management of IRAE suggest that patients receiving ICPi with sCr increase two to three times of baseline, should have ICPi held and receive 0.5 to $1 \mathrm{mg} / \mathrm{kg}$ /day prednisone equivalents (with an increase to $1-2 \mathrm{mg} / \mathrm{kg} /$ day if worsening or no improvement). ${ }^{27}$ These guidelines also state that 'reflex kidney biopsy should be discouraged until corticosteroid treatment has been attempted'. In our cohort, the majority of patients with AKI had ICPi suspended and received corticosteroids (both in those with and without nephrologistconfirmed ICPi nephrotoxicity); however, the given the significant proportion of patients with non-AIN forms of nephrotoxicity, kidney biopsy may be informative in many patients and should be considered strongly in patients with features atypical for AIN (ie, presence of high grade proteinuria and active urinary sediment with absence of white blood cells in the urine), or non-responsive to ICPi suspension and short corticosteroid trial. Izzedine et $a l^{7}$ recently described 12 cases of biopsy-confirmed nephrotoxicity from a cohort of 676 pembrolizumabtreated patients of whom five had ATN, four had AIN, one had MCD and ATN, and one had MCD alone. Pembrolizumab withdrawal and corticosteroid therapy was the most common treatment; however, given the proportion of patients with ATN, which is unlikely to be responsive to corticosteroid therapy, the accompanying editorial suggested an approach of 'diagnosis first, treatment later' ${ }^{28}$ Although our cohort did not demonstrate a significant proportion with ATN, the presence of cases with TMA and MCD/MN support use of an early biopsy strategy in selected patients.

With respect to clinical features, our cohort presented similarly to that of the case series by Izzedine et $a l^{7}$ Mamlouk $e t a l^{8}$ and the recent large multicenter study by Cortazar et al. ${ }^{29}$ Median time from ICPi start to AKI in our cohort was 30 (IQR 21 to 79) days, which compares to 98 (range: 42 to 392) days in Mamlouk et $a l^{8}$ and 270 (range: 30 to 720 ) days in Izzedine et $a l^{7}{ }^{7}$ suggesting that treatment duration before toxicity is variable and that suspicion for kidney IRAE is appropriate, even in patients who have been established on ICPi therapy. The majority of AKI in our cohort was of mild severity and most patients had recovery in kidney function. These findings are congruent with Izzedine $e t a l$, who reported a mean sCr of $2.1 \mathrm{mg} / \mathrm{dL}(185 \mathrm{umol} / \mathrm{L})(\mathrm{SD}: 0.7 \mathrm{mg} / \mathrm{dL}$ $(63 \mathrm{umol} / \mathrm{L}))$ and six out of 12 patients had recovery of $\sim 50 \%$ of their kidney function. Mamlouk et al reported a higher severity of AKI (with 12 out of 16 having AKI grade 3 or higher injury using Acute Kidney Injury Network ${ }^{30}$ classification), but still observed that the majority of patients experienced full or partial kidney recovery. Similarly, in the multicenter cohort of Cortazar et al, $40 \%$ of patients had complete renal recovery; however, it should be noted that the inclusion criteria of this study required that patients have KDIGO stage 2 or greater AKI severity. ${ }^{29}$ This difference in inclusion criteria may also account for the smaller proportion of glomerular disease seen in this cohort, as many patients with glomerular nephrotoxicity may present primarily with proteinuria and only modest (or no change) in sCr.

In our cohort, ICPi was stopped initially in all patients with nephrologist-confirmed nephrotoxicity. This practice is similar to that observed in the studies by Mamlouk et $a l^{8}$ and Izzedine $e t a l^{7}$ with 15 of 16 and 10 of 12 stopping ICPi, respectively. In the larger cohort of Cortazar et al, 31 patients underwent re-challenge with ICPi, with 23\% experiencing recurrent AKI. ${ }^{29}$ This is higher than the estimated recurrence rate that we observed in our cohort; however, the number of patients receiving re-challenge in our study is admittedly small, with few who had undergone kidney biopsy. Also, as inclusion criteria of Cortazar et al required KDIGO stage 2 AKI or greater severity, one may expect that higher recurrence rates would be observed. Additional data on the outcomes of patients with continuation or re-challenge with ICPi are needed.

With respect to risk factors for AKI in our cohort, the presence of other 'non-kidney' IRAE was associated with AKI in both univariable and multivariable analyses (and this association persisted in our sensitivity analysis that excluded AKI episodes that were likely to be related to non-ICPi causes). Non-kidney IRAE may reflect the degree of immune system activation by ICPi, and therefore increase the likelihood of off-target immune effects within the kidney. Some IRAE (eg, colitis and myocarditis) may also mediate AKI through indirect mechanisms such as volume depletion or hemodynamic insult. The association between other IRAE and nephrotoxicity was not assessed in the study by Cortazar $e t a l,{ }^{29}$ but in their cohort, it was observed that $43 \%$ of those with AKI had another non-renal IRAE. Hypertension was also associated with AKI, as has been observed in the broader population with cancer, ${ }^{23}$ and this likely reflects the presence of vascular disease in these patients and the propensity for kidney injury through non-immune means. Notably, in our sensitivity analysis (that excluded AKI events presumed secondary to pre-renal and hemodynamic insults), this association was no longer significant. We did not observe an association between co-prescribed drugs (including PPI, NSAIDs, and antibiotics) and AKI risk. Previous series have hypothesized that receipt of ICPi may modify immune tolerance to these drugs and that such agents should be discontinued after ICPi-associated AIN (and potentially prior to re-challenge with ICPi). ${ }^{20} 31$ Seethapathy et al also assessed the possible association between PPI use and AKI in ICPi recipients, and they did observe an association between PPI use and sustained AKI (eg, persistent creatinine elevation for $\geq 3$ days), but not 
all ICPi nephrotoxicity. ${ }^{21}$ While this association was not observed in our cohort, this may well be related to the inclusion of both confirmed immune-mediated episodes of AKI as well as non-confirmed episodes of AKI. Moreover, the small proportion of individuals receiving these agents may have precluded detection of such an association. As in our study, Seethapathy $e t a l^{21}$ and Cortazar $e t$ $a l^{29}$ did not observe association between NSAID use and AKI. Finally, we did not observe a difference in survival in those with and without AKI during ICPi therapy. This may be due to the fact that the cohort included predominantly patients with advanced cancer in whom ICPi treatment was indicated, with substantial baseline mortality risk. Also, the majority of AKI in our cohort was mild, with recovery of kidney function, as such, AKI and progression to CKD may have been less likely to contribute to subsequent mortality.

It should be noted that our cohort predominantly included patients with normal baseline kidney function. This may be owing to the fact that many patients may have been participants in clinical trials, and many trials of cancer therapy exclude patients with CKD and ESKD. ${ }^{32}$ As such, additional data is needed to address AKI associated with ICPi in the CKD population, as well as its impact on both renal and cancer outcomes. This may become increasingly important as many patients with CKD and cancer may be candidates for immunotherapies as their indications broaden.

Our study has several strengths, including a comprehensive assessment of AKI among ICPi recipients using sCr data. We were able to include all recipients of ICPi in our center (through identification from an electronic ordering system) and were therefore able to provide an estimate as to AKI incidence in this population. We also were able to perform a detailed chart review to reliably assess cancer and ICPi treatment characteristics, as well as comorbidities and co-prescriptions to evaluate AKI risk factors. In addition, we had a substantial proportion of patients with nephrologist- or biopsy-confirmed nephrotoxicity with description of varied kidney lesions, including glomerular disease.

Our study has several important limitations. Most of the AKI episodes were neither confirmed nor treated by a nephrologist (as may be commonplace among patients managed by medical oncologists). As such, the definite cause of AKI could not be ascertained in all cases. As result, we were able to evaluate overall AKI risk factors, but not necessarily factors specific to immune-mediated ICPi nephrotoxicity. However, we did find that, even in patients not assessed by a nephrologist, a substantial proportion were receiving steroid therapy at the time of AKI, suggesting that in many patients, immunemediated injury may have been suspected and empiric glucocorticoids administered. We also sought to address this issue in our sensitivity analysis (via the exclusion of AKI presumed to be related to non-ICPi causes). In this model, hypertension was no longer significantly associated with AKI, which likely speaks to its mediation of
AKI via hemodynamic mechanisms, rather than immune means. The relatively small number of biopsy-confirmed cases of ICPi nephrotoxicity at single center (particularly in view of current ASCO guidelines ${ }^{27}$ recommendations regarding kidney biopsy), makes such risk factor analysis a challenge. In particular, the small number of events allowed for inclusion of only a limited number of covariates in multivariable logistic regression. The majority of patients $(85 \%)$ had advanced melanoma which may limit generalizability of our findings. Moreover, melanoma is not commonly associated with AKI, ${ }^{22}$ therefore we believe that ICPi treatment likely played an important role in the development of this complication. Also, the majority of patients were receiving ipilimumab monotherapy, which may be less representative than use of single agent PD-1/ PD-L1 therapy (currently more frequent among ICPitreated cancers); however, in a recent meta-analysis of IRAE in melanoma patients treated with these agents, AKI events were not reported to differ between the CTLA-4 and PD-1 agents, ${ }^{33}$ and as such, our findings likely have implications with respect to both classes. Finally, we had a limited number of patients who received re-challenge with ICPi after AKI (with even fewer that had undergone kidney biopsy) and this may have limited our ability to estimate recurrence rates. Larger cohorts are needed to better characterize both AKI and ICPi nephrotoxicity specifically, in this population.

\section{CONCLUSION}

Overall, AKI is common complication in patients receiving ICPi treatment. As such, distinguishing kidney IRAE from other causes of AKI will present a frequent challenge to oncology and nephrology practitioners. ICPi-associated nephrotoxicity includes AKI due to AIN/ ATI or ATN as well as glomerulopathies such as kidneylimited TMA, MCD, and MN. Indeed, the potential for glomerular disease, and kidney lesions which may not benefit from corticosteroids, suggests that kidney biopsy should be very strongly considered when ICPi-associated nephrotoxicity is suspected.

Most AKI in this population was of mild-to-moderate severity, and responsive to temporarily stopping ICPi and systemic corticosteroids for severe cases. There was no signal for harm when patients who developed AKI were re-challenged with ICI. Re-challenge with ICPi can be considered in such patients, particularly those with limited therapeutic options. The presence of other IRAE may be associated with AKI and should raise clinical suspicion of ICPi nephrotoxicity. However, these patients are not exempted from other causes of AKI such as volume contraction, ischemic tubular injury, obstructive nephropathy, and nephrotoxicity from concomitant cancer treatments. A careful evaluation must be performed in every cancer patient with ICPi-associated AKI, including urinary microscopy and kidney biopsy in cases with severe AKI or features atypical for AIN. 
Twitter Alejandro Meraz-Muñoz @Nephroguy and Abhijat Kitchlu @onconeph

Contributors Drs Meraz-Munoz, Amir and Kitchlu had full access to all of the data in the study and take responsibility for the integrity of the data and the accuracy of the data analysis. Concept and design: Kitchlu, Chan, Amir, Kim and Wald. Acquisition, analysis, or interpretation of data: Meraz-Munoz, Ragobar and Amir. Drafting of the manuscript: Meraz-Munoz, Kitchlu, Amir and Wald. Critical revision of the manuscript for important intellectual content: All authors. Statistical analysis: Meraz-Munoz, Kitchlu and Amir. Administrative, technical or material support: Kitchlu, Amir and Chan. Supervision: Kitchlu, Amir and Wald.

Funding The authors have not declared a specific grant for this research from any funding agency in the public, commercial or not-for-profit sectors.

Competing interests None declared.

Patient consent for publication Not required.

Ethics approval This study was approved by the Research Ethics Board of the University Health Network, Toronto, Canada.

Provenance and peer review Not commissioned; externally peer reviewed.

Data availability statement Data are available upon reasonable request.

Open access This is an open access article distributed in accordance with the Creative Commons Attribution Non Commercial (CC BY-NC 4.0) license, which permits others to distribute, remix, adapt, build upon this work non-commercially, and license their derivative works on different terms, provided the original work is properly cited, appropriate credit is given, any changes made indicated, and the use is non-commercial. See http://creativecommons.org/licenses/by-nc/4.0/.

\section{ORCID iDs}

Alejandro Meraz-Muñoz http://orcid.org/0000-0002-8174-1235

Abhijat Kitchlu http://orcid.org/0000-0002-4340-5046

\section{REFERENCES}

1 Sury K, Perazella MA, Shirali AC. Cardiorenal complications of immune checkpoint inhibitors. Nat Rev Nephrol 2018;14:571-88.

2 Postow MA, Sidlow R, Hellmann MD. Immune-Related adverse events associated with immune checkpoint blockade. N Engl J Med 2018;378:158-68.

3 Shingarev R, Glezerman IG. Kidney complications of immune checkpoint inhibitors: a review. Am J Kidney Dis 2019;74:529-37.

4 Cortazar FB, Marrone KA, Troxell ML, et al. Clinicopathological features of acute kidney injury associated with immune checkpoint inhibitors. Kidney Int 2016;90:638-47.

5 Shirali AC, Perazella MA, Gettinger S. Association of acute interstitial nephritis with programmed cell death 1 inhibitor therapy in lung cancer patients. Am J Kidney Dis 2016;68:287-91.

6 Kitchlu A, Fingrut W, Avila-Casado C, et al. Nephrotic Syndrome With Cancer Immunotherapies: A Report of 2 Cases. Am J Kidney Dis 2017;70:581-5.

7 Izzedine H, Mathian A, Champiat S, et al. Renal toxicities associated with pembrolizumab. Clin Kidney J 2019;12:81-8.

8 Mamlouk O, Selamet U, Machado S, et al. Nephrotoxicity of immune checkpoint inhibitors beyond tubulointerstitial nephritis: single-center experience. J Immunother Cancer 2019;7:2.

9 Weber JS, Hodi FS, Wolchok JD, et al. Safety profile of nivolumab monotherapy: a pooled analysis of patients with advanced melanoma. J Clin Oncol 2017;35:785-92.

10 Abdel-Wahab N, Shah M, Suarez-Almazor ME. Adverse events associated with immune checkpoint blockade in patients with cancer: a systematic review of case reports. PLoS One 2016;11:e0160221:07-29.

11 Weber J, Mandala M, Del Vecchio M, et al. Adjuvant nivolumab versus ipilimumab in resected stage III or IV melanoma. $N$ Engl $\mathrm{J}$ Med 2017;377:1824-35.

12 Sznol M, Ferrucci PF, Hogg D, et al. Pooled analysis safety profile of nivolumab and ipilimumab combination therapy in patients with advanced melanoma. J Clin Oncol 2017;35:3815-22.

$13 \mathrm{KDIGO}$ clinical practice guideline for acute kidney injury. Kidney Int Suppl 2012;2:1.

14 Gupta S, Cortazar FB, Riella LV, et al. Immune checkpoint inhibitor nephrotoxicity: update 2020. Kidney360 2020;1:130-40.

15 Levey AS, Stevens LA, Schmid CH, et al. A new equation to estimate glomerular filtration rate. Ann Intern Med 2009;150:16.

16 Lam AQ, Humphreys BD. Onco-nephrology: AKI in the cancer patient. Clin J Am Soc Nephrol 2012;7:1692-700.

17 Rosner $\mathrm{MH}$, Perazella MA. Acute kidney injury in patients with cancer. Ingelfinger JR, editor. N Engl J Med 2017;376:1770-81.

18 Mason NT, Khushalani NI, Weber JS, et al. Modeling the cost of immune checkpoint inhibitor-related toxicities. JCO 2016;34:6627.

19 Hirsch J. Incidence of AKI in immune checkpoint inhibitors, single center study [Abstract]. J Am Soc Nephrol 2016;26:761.

20 Wanchoo R, Karam S, Uppal NN, et al. Adverse renal effects of immune checkpoint inhibitors: a narrative review. Am J Nephrol 2017;45:160-9.

21 Seethapathy $\mathrm{H}$, Zhao S, Chute DF, et al. The incidence, causes, and risk factors of acute kidney injury in patients receiving immune checkpoint inhibitors. Clin J Am Soc Nephrol 2019;14:1692-700.

22 Christiansen CF, Johansen MB, Langeberg WJ, et al. Incidence of acute kidney injury in cancer patients: a Danish population-based cohort study. Eur J Intern Med 2011;22:399-406.

23 Kitchlu A, McArthur E, Amir E, et al. Acute kidney injury in patients receiving systemic treatment for cancer: a population-based cohort study. J Natl Cancer Inst 2018;11:727-36.

$24 \mathrm{Kim}$ Y. Relapse of membranous nephropathy with cancer immunotherapy. Clin Kidney J 2019:1-3.

25 Abudayyeh A, Abdelrahim M, Selamet U, et al. Checkpoint inhibitor induced glomerulonephritis. JCO 2018;36:e15083.

26 Fadel F, El Karoui K, Knebelmann B. Anti-Ctla4 antibody-induced lupus nephritis. N Engl J Med 2009;361:211-2.

27 Brahmer JR, Lacchetti C, Schneider BJ, et al. Management of immune-related adverse events in patients treated with immune checkpoint inhibitor therapy: American Society of clinical oncology clinical practice guideline. J Clin Oncol 2018;36:1714-68.

28 Sprangers B. Pembrolizumab-related renal toxicities: diagnosis first, treatment later. Clin Kidney J 2019;12:78-80.

29 Cortazar FB, Kibbelaar ZA, Glezerman IG, et al. Clinical features and outcomes of immune checkpoint inhibitor-associated AKI: a multicenter study. J Am Soc Nephrol 2020;31:435-46.

30 Mehta RL, Kellum JA, Shah SV, et al. Acute kidney injury network: report of an initiative to improve outcomes in acute kidney injury. Crit Care 2007;11:R31.

31 Perazella MA, Shirali AC. Immune checkpoint inhibitor nephrotoxicity: what do we know and what should we do? Kidney Int 2020;97:62-74.

32 Kitchlu A, Shapiro J, Amir E, et al. Representation of patients with chronic kidney disease in trials of cancer therapy. JAMA 2018;319:2437.

33 Almutairi AR, McBride A, Slack M, et al. Potential immune-related adverse events associated with monotherapy and combination therapy of ipilimumab, nivolumab, and pembrolizumab for advanced melanoma: a systematic review and meta-analysis. Front Oncol 2020;10:91. 